\title{
A Case of Eosinophilic Esophagitis Associated with Herpes Esophagitis in a Pediatric Patient
}

\author{
Jisun Kim${ }^{1}$, Kunsong Lee ${ }^{1}$ and Wonae Lee ${ }^{2}$ \\ Department of ${ }^{1}$ Pediatrics, ${ }^{2}$ Pathology, Dankook University College of Medicine, Cheonan, Korea
}

Eosinophilic esophagitis is a rare disease in Asian countries, but its incidence is growing rapidly in Western countries. The main pathophysiology of eosinophilic esophagitis is esophageal epithelial barrier dysfunction; disruption of the esophageal epithelial barrier easily induces antigen sensitization to foods and aeroallergens, which leads to subsequent esophageal inflammation as a result of eosinophil recruitment. Here we report a case of an 11-year-old Korean boy who suffered from fever, odynophagia, dysphagia, and chest pain. His upper endoscopic findings showed longitudinal ulcers with a volcano-like appearance at the distal esophagus. Polymerase chain reaction test results and biopsy specimens were positive for herpes simplex virus type 1 . He was treated with acyclovir and a proton pump inhibitor, but his follow-up endoscopy showed typical patterns of eosinophilic esophagitis, and the biopsy specimens were compatible with the diagnostic criteria for eosinophilic esophagitis. Therefore, we report a very rare case of eosinophilic esophagitis after herpes esophagitis in a Korean child with normal immunity. Clin Endosc 2019;52:606-611

Key Words: Child; Eosinophilic esophagitis; Herpes simplex virus

\section{INTRODUCTION}

Eosinophilic esophagitis (EoE) is defined as an immuneand antigen-mediated chronic esophageal inflammatory disease with eosinophilic accumulation and esophageal dysfunction symptoms, such as vomiting, dysphagia, and food impactions. ${ }^{1}$ Although the incidence of EoE is rapidly increasing in Europe and the United States, EoE is a very rare disease in Korea and there have been limited studies on EoE in Korea and other Asian countries. ${ }^{2,3}$ The occurrence of EoE is associated with abnormal esophageal epithelial barrier function, and collapse of the esophageal epithelium is one of the most important factors in the development of EoE. ${ }^{1,4}$ This abnor-

Received: December 31, 2018 Revised: May 3, 2019

Accepted: May 8, 2019

Correspondence: Kunsong Lee

Department of Pediatrics, Dankook University College of Medicine, 119 Dandae-ro, Dongnam-gu, Cheonan 31116, Korea

Tel: +82-41-550-3968, Fax: +82-41-550-3949, E-mail: pdlks@dankook.ac.kr ORCID: https://orcid.org/0000-0001-7318-2296

(cc) This is an Open Access article distributed under the terms of the Creative Commons Attribution Non-Commercial License (http://creativecommons.org/ licenses/by-nc/3.0) which permits unrestricted non-commercial use, distribution, and reproduction in any medium, provided the original work is properly cited. mality can easily lead to sensitization to food and environmental antigens, followed by cytokine mediated recruitment of eosinophils to the esophagus. ${ }^{1}$

Herpes simplex virus (HSV) is a common cause of infectious esophagitis in immunocompromised patients, but HSV esophagitis is also found in immunocompetent healthy children and adults. ${ }^{5-8}$ HSV infection in the esophagus can induce the breakdown of the esophageal mucosa and activate an immune reaction that sensitizes the esophagus to foods and aeroallergens. ${ }^{5}$ In practice, there have been reports of subsequent occurrences of EoE due to HSV infection. ${ }^{5-7,9-12}$ However, active EoE can also cause HSV esophagitis as a result of active untreated EoE disrupting the esophageal mucosa integrity and allowing infectious agents, such as HSV, to invade the esophageal mucosa more easily. ${ }^{10}$

The patient described in this report was diagnosed with EoE after esophageal epithelial damage due to HSV esophagitis. Until now, EoE has not been diagnosed after HSV esophagitis in Korea; we report a case where EoE was diagnosed after HSV esophagitis in an immunocompetent pediatric patient, and we present a review of relevant literature on the subject. 


\section{CASE REPORT}

An 11-year-old boy who had allergic rhinitis without other medical problems was admitted with fever, odynophagia, dysphagia, and chest pain. On examination, his body temperature was $38.2^{\circ} \mathrm{C}$ and he had multiple ulcers on his lip and hard palate. The initial esophagogastroduodenoscopy (EGD) (Olympus GIF-Q260 Video Gastroscope; Olympus, Tokyo, Japan) revealed numerous depressed linear ulcerative lesions at the mid-esophagus. The longitudinal ulcers had erythematous mucosa with raised margins and yellow bases with a volcano-like appearance at the distal esophagus (Fig. 1A). Polymerase chain reaction (PCR) and immunohistochemistry were performed with the EGD biopsy specimens in order to identify the causative infection of the ulcers.

We extracted genomic DNA from the fresh esophageal biopsy tissues using the QIAsymphony kit (Qiagen, Hombrechtikon, Switzerland), following preparation with tissue lysis buffer and proteinase K solution (Qiagen). We mixed the primer mixture of HSV type 1 and type 2 (BioCore, Seoul, Korea) and the genomic DNA. PCR was performed with a cycling condition consisting of a denaturation step for $12 \mathrm{~min}$ utes $\left(95^{\circ} \mathrm{C}\right)$, followed by 35 cycles of 45 seconds at $94^{\circ} \mathrm{C}, 67^{\circ} \mathrm{C}$, and $72^{\circ} \mathrm{C}$ respectively, and a final extension at $72^{\circ} \mathrm{C}$ for 5 minutes. Electrophoresis was performed with the PCR products and the internal control. The marker of the 279 base pair was HSV type 1 and the 180 base pair was HSV type 2. Although HSV type I was positive in the PCR test, HSV was negative in the immunohistochemistry exam, which also showed nonspecific inflammatory and ulcerative findings without cytopathic changes and intranuclear inclusions (Fig. 1B). We performed PCR with skin, blood, and gastrointestinal tissues of six patients in parallel. HSV type 1 was only positive in the esophageal tissue of this patient, and so we could exclude the possibility of contamination during the test. Cytomegalovirus and Epstein-Barr virus were negative in the PCR test, and there was no evidence of Candida infection in the histopathologic stain. We made a diagnosis of HSV esophagitis, and treated the patient with intravenous acyclovir ( $5 \mathrm{mg} / \mathrm{kg} /$ day $)$ and a proton pump inhibitor (PPI) (1 mg/kg/day). The symptoms improved and we performed an EGD 9 days after admission.

Although the mucosal edema and erythema were improved, the ulcers that were observed in the mid and distal esophagus remained as scars (Fig. 2A). Histopathologic examination showed an improvement in inflammation and ulcerative change, and HSV type 1 was no longer detectable by PCR (Fig. 2B). As a result of these improvements, the patient was discharged 11 days after admission. Investigations to assess immune function, such as measurement of immunoglobulins, complement, and lymphocyte subsets, were normal, and an antibody test for human immunodeficiency virus was negative. The total IgE was increased to $512.7 \mathrm{IU} / \mathrm{mL}$ (1.9-170.0), and intravenous acyclovir and PPI were administered for 10 days. The intravenous PPI treatment was then changed to an oral medication and used for a total of 8 weeks.

We performed a follow-up EGD at 12 weeks after diagnosis,
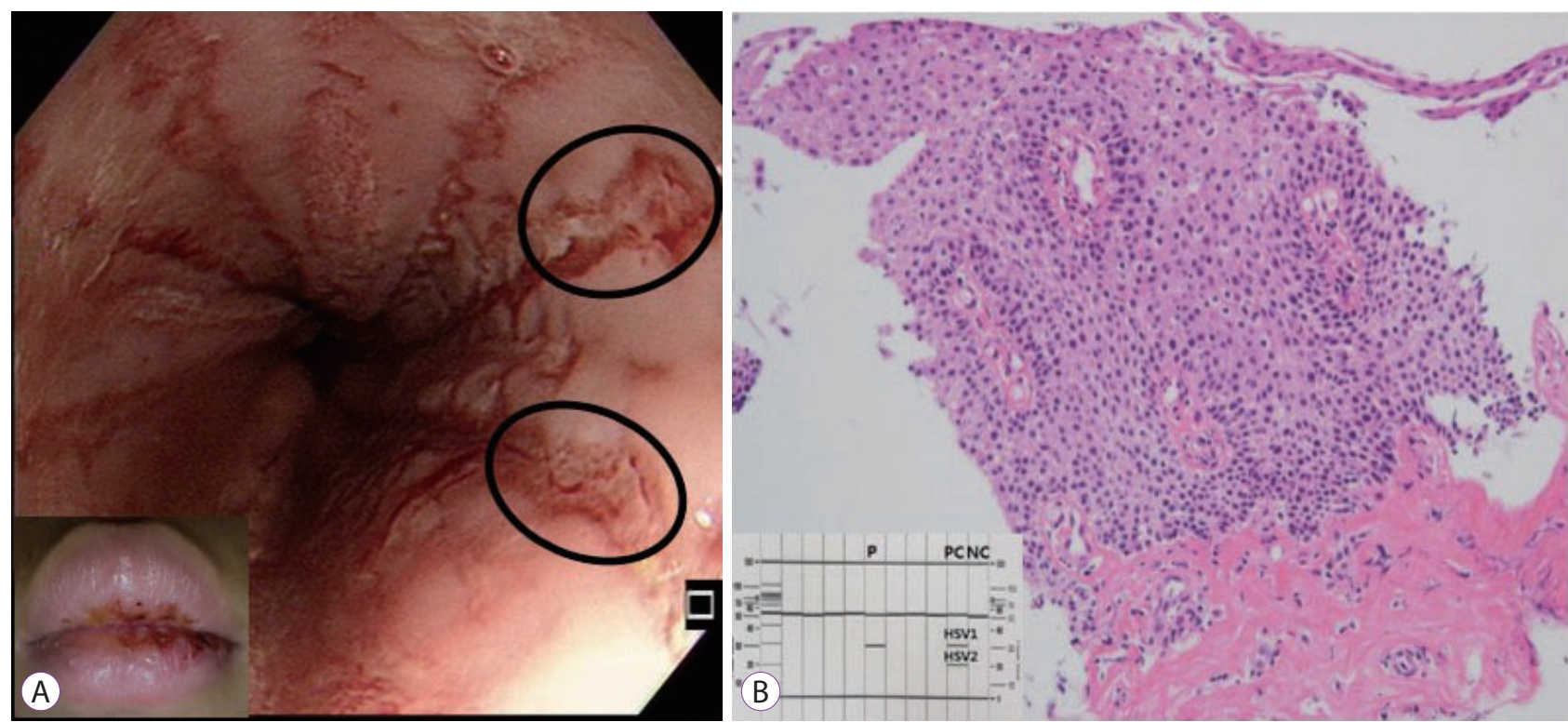

Fig. 1. (A) Longitudinal ulcers with raised margins, yellow bases with a volcano-like appearance on the distal esophagus (black ellipses), and ulcers on the lip are shown. (B) The initial esophageal biopsy specimen shows nonspecific inflammatory and ulcerative findings without cytopathic changes or intranuclear inclusions (hematoxylin and eosin stain, $\times 200$ ). The polymerase chain reaction test is positive for herpes simplex virus (HSV) 1; patient (P), positive control (PC), and negative control (NC). 
in which endoscopic findings of EoE with white exudates and multiple linear furrows without mucosal blood vessels in the mid and distal esophagus were identified (Fig. 3A, B). Upon histopathologic examination of the endoscopic biopsies, there was no evidence of HSV infection, and large quantities of intraepithelial eosinophils were observed in the proximal, middle, and distal regions of the esophagus (Fig. 3C). In total, 20, 50 , and 200 eosinophils were observed in high-power fields of the proximal middle, and distal regions of the esophagus, respectively. In the stomach and duodenum, 1 and 15 eosinophils were identified in high-power fields, respectively, and the total blood eosinophil count was increased to $500 / \mathrm{mm}^{3}$. HSV
IgG was converted from negative in the initial test, to positive at follow-up, but IgM was negative in both tests.

Although we treated with intravenous acyclovir and PPI for 10 days and oral PPI for the next 7 weeks, the patient's endoscopic and histological findings were compatible with EoE; as such, we made a diagnosis of EoE following HSV esophagitis. Although the patient's chest pain and odynophagia resolved after treatment, he still experienced epigastric pain with meals. We intend on starting a six food elimination diet followed by a topical steroid if a food elimination diet confirms intolerance.
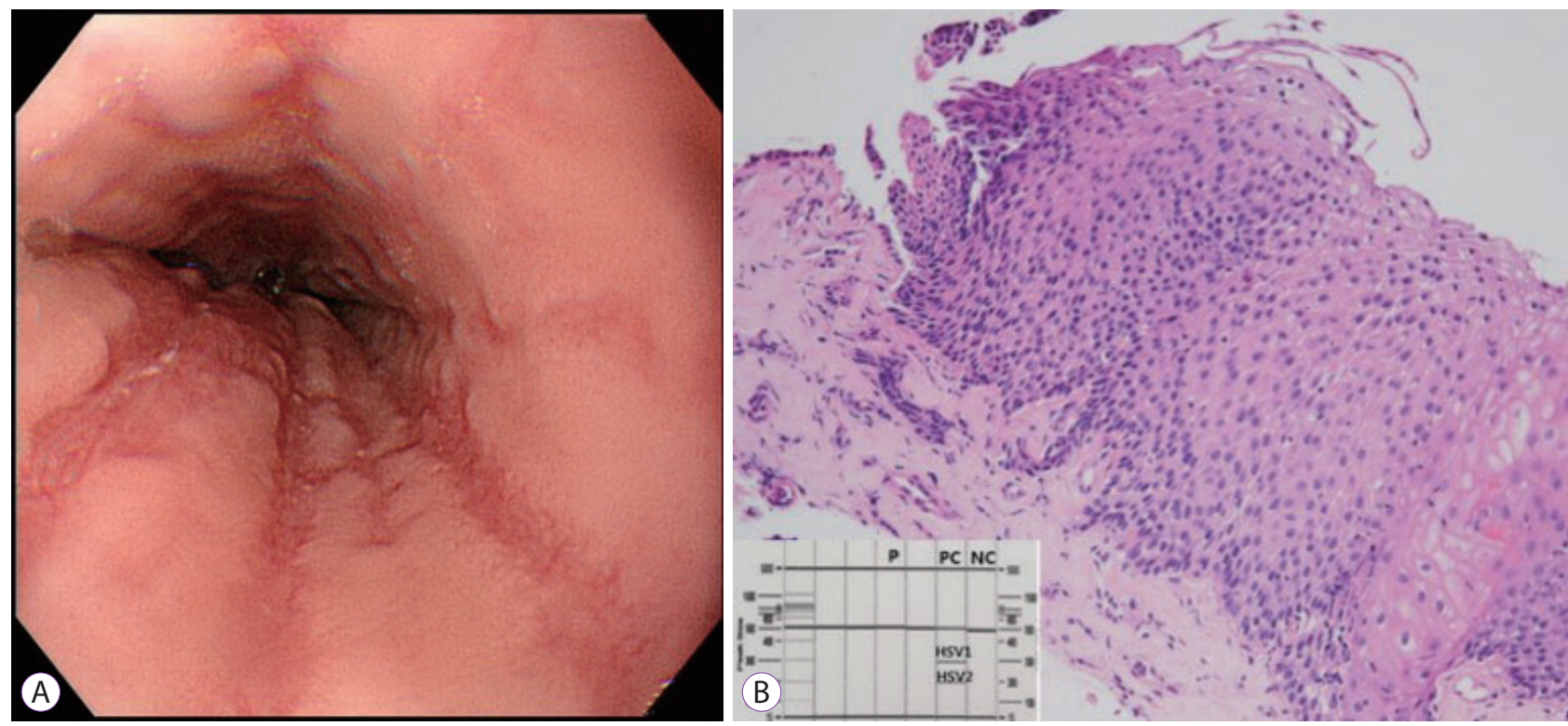

Fig. 2. (A) After acyclovir treatment, although the middle and distal esophagus shows improvement in mucosal edema and ulcers, the distal esophagus still shows ulceration with scarring. (B) The post-treatment biopsy specimen shows an improvement in inflammation, and the herpes simplex virus (HSV) type 1 polymerase chain reaction test shows a negative change. P, patient; PC, positive control; NC, negative control.
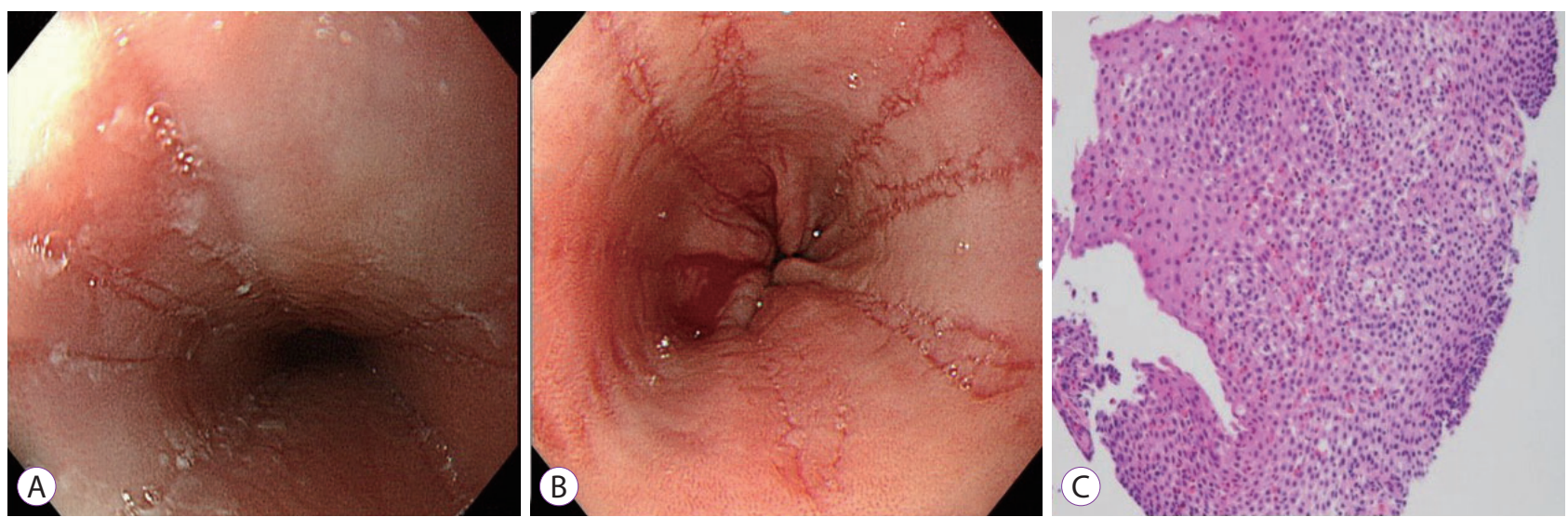

Fig. 3. (A) The follow-up endoscopy after acyclovir and proton pump inhibitor treatment shows multiple white exudates and moderate mucosal edema with furrows in the proximal esophagus. (B) The distal esophagus shows multiple longitudinal furrows with loss of mucosal vascularity. (C) The distal esophageal biopsy specimen from the follow-up endoscopy shows massive intraepithelial eosinophilic infiltration (up to 200 per high power field) and subepithelial fibrosis (hematoxylin and eosin stain, $\times 200$ ). 


\section{DISCUSSION}

Currently, the incidence of EoE is approximately 10 cases per 100,000 persons worldwide, and Caucasians are more affected by EoE than any other race. ${ }^{3,13}$ Asian countries have not undergone population-based studies of EoE because of the rareness of the disease in Asians. Indeed, Kim et al. ${ }^{14}$ reported that in a single center study in Korea the percentage was $0.23 \%$ among patients who received EGD over an 8 year period. There have been no studies on pediatric EoE in Korea.
The presented case is significant because of the rarity of EoE following HSV esophagitis in Korea.

EoE is characterized by esophageal dysfunction and esophageal eosinophilia due to complex mechanisms, such as genetic and environment factors, and antigenic stimuli from food or aeroallergens. ${ }^{4}$ This immune response is mediated by $\mathrm{T}$ helper 2 (Th2) lymphocytes, which secrete various cytokines that are responsible for eosinophil recruitment into the esophagus, and subsequent disruption of the epithelial barrier. ${ }^{4}$ The impaired esophageal barrier is one of the known factors asso-

Table 1. Clinical Features of Previously Reported Cases Associated with Eosinophilic Esophagitis and Herpes Simplex Virus Esophagitis

\begin{tabular}{|c|c|c|c|c|c|c|}
\hline Case & Sex & $\begin{array}{l}\text { Age at diagno- } \\
\text { sis of EoE ( } y r)\end{array}$ & Nation & Past medical history & $\begin{array}{c}\text { Order of HSV infection } \\
\text { and EoE }\end{array}$ & $\begin{array}{c}\text { Endoscopic finiding at } \\
\text { presentation }\end{array}$ \\
\hline 1 & $\mathrm{M}$ & 14 & Australia & Asthma & $\begin{array}{l}\text { HSV infection precede } \\
8 \mathrm{wk}\end{array}$ & $\begin{array}{l}\text { Longitudinal furrows and } \\
\text { linear ulcers }\end{array}$ \\
\hline 2 & M & 16 & Australia & $\begin{array}{c}\text { Caustic esophagitis, } \\
\text { Asthma, and FA (legumes) }\end{array}$ & $\begin{array}{l}\text { HSV infection precede } \\
6 \mathrm{wk}\end{array}$ & Longitudinal furrows \\
\hline 3 & M & 9 & Brazil & Atopic dermatitis & $\begin{array}{l}\text { HSV infection precede } \\
4 \mathrm{wk}\end{array}$ & Unknown \\
\hline 4 & M & 20 & Portugal & Asthma & $\begin{array}{l}\text { HSV infection precede } \\
6 \mathrm{wk}\end{array}$ & Linear erosions \\
\hline 5 & M & 4 & USA & No & $\begin{array}{l}\text { HSV infection precede } \\
6 \mathrm{wk}\end{array}$ & Unkown \\
\hline 6 & $\mathrm{~F}$ & 17 & Croatia & FA (milk) & Concurrent & $\begin{array}{l}\text { Longitudinal furrow with } \\
\text { concentric rings }\end{array}$ \\
\hline 7 & M & 18 & USA & AR and FA (wheat) & Concurrent & Shallow round ulcers \\
\hline 8 & $\mathrm{~F}$ & 25 & Switzerland & Unknown & EoE precede unknown years & White exudates \\
\hline 9 & $\mathrm{~F}$ & 22 & Switzerland & Unknown & EoE precede 5 yr & $\begin{array}{l}\text { Longitudinal furrows and } \\
\text { decreased vascular pattern }\end{array}$ \\
\hline 10 & M & 29 & Switzerland & Unknown & Concurrent & $\begin{array}{l}\text { Furrows, white exudates, } \\
\text { solitary ulcer }\end{array}$ \\
\hline 11 & M & 24 & Switzerland & Unknown & EoE precede 6 yr & $\begin{array}{l}\text { Erosions and puncuate } \\
\text { ulcerations }\end{array}$ \\
\hline 12 & M & 28 & Switzerland & Asthma, AR & $\begin{array}{l}\text { HSV infection precede } \\
9 \mathrm{wk}\end{array}$ & White exudate and edema \\
\hline 13 & $\mathrm{M}$ & 13 & USA & No & $\begin{array}{l}\text { HSV infection precede } \\
4 \mathrm{wk}\end{array}$ & Furrows, erythema \\
\hline 14 & M & 21 & Spain & No & $\begin{array}{l}\text { HSV infection precede } \\
12 \mathrm{wk}\end{array}$ & Furrows and white exudates \\
\hline 15 & $\mathrm{M}$ & 8 & USA & Asthma & $\begin{array}{l}\text { HSV infection precede } \\
6 \mathrm{wk}\end{array}$ & $\begin{array}{l}\text { Erosions and purulent exu- } \\
\text { date }\end{array}$ \\
\hline 16 & M & 14 & USA & Atopic dermatitis & $\begin{array}{l}\text { HSV infection precede } \\
8 \mathrm{wk}\end{array}$ & Edema, furrows, and rings \\
\hline 17 & M & 8 & USA & Asthma & $\begin{array}{l}\text { HSV infection precede } \\
8 \mathrm{wk}\end{array}$ & Edema and furrows \\
\hline 18 & M & 11 & USA & Asthma & $\begin{array}{l}\text { HSV infection precede } \\
20 \mathrm{wk}\end{array}$ & Edema and furrows \\
\hline
\end{tabular}

AR, allergic rhinitis; EoE, eosinophilic esophagitis; FA, food allergy; HSV, herpes simplex virus. 
ciated with the occurrence of EoE.

HSV esophagitis is common in immunocompromised patients, but has also been continuously reported in immunocompetent adult and pediatric patients. ${ }^{8}$ As in this case, patients with HSV esophagitis present with retrosternal pain and odynophagia with or without fever. ${ }^{11}$ Endoscopic findings of HSV esophagitis demonstrate circumscribed ulcers with raised edges and erythema, described as volcano-like ulcers. ${ }^{11}$ In the histopathological diagnosis of HSV esophagitis, acute inflammation, ulceration, and cytopathic changes are major points. ${ }^{15}$ A definitive histological diagnosis of HSV esophagitis requires herpes virus isolation from the tissue during cell culture. ${ }^{15}$ Although viral culture offers high sensitivity and specificity, transport conditions and laboratory environmental factors have an effect on virus isolation. ${ }^{8}$ In addition, biopsies should be taken from the ulcer margins in order to increase the HSV isolation rate. ${ }^{8}$ On the other hand, the PCR test has a higher detection rate for HSV than cell culture. ${ }^{8}$ Indeed, in the described case, HSV was only detected by PCR and not from immunohistochemical examination. We believe that the histological features of HSV infection were not clearly observed in this case because the site of the biopsies was the base of the ulcer.

The link between EoE and HSV esophagitis has been reported in some cases in Western countries. ${ }^{5-7,10-12,16-18}$ There has been debate as to whether HSV esophagitis is the causative agent of EoE, or whether EoE is a predisposing factor for HSV esophagitis. Esophageal tissue injury due to HSV infection may cause a breakdown of the esophageal mucosal barrier, resulting in the development of hyper-reactivity to food and environmental antigens. ${ }^{11,12}$ This change may also trigger Th2 cell clones, which induce cytokine secretion and eosinophilic inflammation. ${ }^{11}$ Additionally, EoE might already be present prior to HSV infection, because the esophageal eosinophilic mucosal inflammation in EoE is susceptible to candidiasis, HSV, and other viral infections. ${ }^{11}$ In this case, HSV infection occurred first, followed by EoE. We found 18 cases related to EoE and HSV esophagitis reported in PubMed Central (Table 1); in these cases, the mean age of EoE occurrence was $16.7 \pm 7.3$ years (4-29 years), and HSV infection preceded EoE in 12 of 18 cases. HSV esophagitis and EoE were simultaneously detected in three cases, and in the remaining three cases, HSV esophagitis was diagnosed following EoE. However, it is difficult to clearly identify the relationship between EoE and HSV esophagitis. Acute inflammation as a result of HSV infection can induce pathologic findings that are dominated by neutrophils, and EoE may be masked if there is an underlying disease. HSV esophagitis is more likely to occur with inflammation of the esophageal mucosa, as in EoE, because HSV esophagitis is rare in individuals with normal immunity.
However, as in this case, there have been reported cases of EoE following HSV infection. In the current case, the child had no previous EoE symptoms, but EoE occurred following HSV esophagitis, with ulceration in the mouth and gingiva. Through several cases that have been previously reported, we found that the mean duration of EoE occurrence following HSV esophagitis was 8 weeks. In the current case, EoE was detected by EGD 12 weeks after the diagnosis of HSV esophagitis.

Thus, we report a case of HSV esophagitis in a healthy child with normal immune function. It is not yet established whether HSV esophagitis is a causative factor for EoE, or whether EoE is a predisposing factor for HSV esophagitis. However, if HSV esophagitis is confirmed, endoscopic follow-up at 8 weeks should be considered in order to establish whether EoE is present.

\section{Conflicts of Interest}

The authors have no financial conflicts of interest.

\section{Acknowledements}

I acknowledge the special education on eosinophilic esophagitis from Professor Glenn Furuta and Nathalie Nguyen from the Children's Hospital Colorado.

\section{REFERENCES}

1. Furuta GT, Katzka DA. Eosinophilic esophagitis. N Engl J Med 2015;373:1640-1648.

2. Warners MJ, de Rooij W, van Rhijn BD, et al. Incidence of eosinophilic esophagitis in the Netherlands continues to rise: 20-year results from a nationwide pathology database. Neurogastroenterol Motil 2018;30:e13165.

3. Moawad FJ. Eosinophilic esophagitis: incidence and prevalence. Gastrointest Endosc Clin N Am 2018;28:15-25.

4. Clayton F, Peterson K. Eosinophilic esophagitis: pathophysiology and definition. Gastrointest Endosc Clin N Am 2018;28:1-14.

5. Fritz J, Lerner D, Suchi M. Herpes simplex virus esophagitis in immunocompetent children: a harbinger of eosinophilic esophagitis? J Pediatr Gastroenterol Nutr 2018;66:609-613.

6. Žaja Franulović O, Lesar T, Busic N, Tešović G. Herpes simplex primo-infection in an immunocompetent host with eosinophilic esophagitis. Pediatr Int 2013;55:e38-e41.

7. Sehgal S, Darbari A, Bader A. Herpes simplex virus and eosinophilic esophagitis. J Pediatr Gastroenterol Nutr 2013;56:e1.

8. Rodrigues F, Brandão N, Duque V, Ribeiro C, António AM. Herpes simplex virus esophagitis in immunocompetent children. J Pediatr Gastroenterol Nutr 2004;39:560-563.

9. Iriarte Rodriguez A, Frago Marquínez I, de Lima Piña GP. A case report: asymptomatic esophageal eosinophilia after herpes simplex esophagitis. Controversies in the therapeutic approach. Rev Esp Enferm Dig 2018;110:471-472.

10. Zimmermann D, Criblez DH, Dellon ES, et al. Acute herpes simplex viral esophagitis occurring in 5 immunocompetent individuals with eosinophilic esophagitis. ACG Case Rep J 2016;3:165-168.

11. Monsanto P, Almeida N, Cipriano MA, Gouveia H, Sofia C. Concom- 
itant herpetic and eosinophilic esophagitis--a causality dilemma. Acta Gastroenterol Belg 2012;75:361-363.

12. Squires KA, Cameron DJ, Oliver M, da Fonseca Junqueira JC. Herpes simplex and eosinophilic oesophagitis: the chicken or the egg? J Pediatr Gastroenterol Nutr 2009;49:246-250.

13. Lee K, Furuta GT, Nguyen N. Eosinophilic esophagitis is an underlying cause for gastrointestinal concerns in children. Front Pediatr 2018;6:113.

14. Kim GH, Jung KW, Jung HY, et al. Diagnostic trends and clinical characteristics of eosinophilic esophagitis: a Korean, single-center database study. J Neurogastroenterol Motil 2018;24:248-254.

15. Al-Hussaini AA, Fagih MA. Herpes simplex ulcerative esophagitis in healthy children. Saudi J Gastroenterol 2011;17:353-356.

16. Cristoforo TA, Rietsma K, Wilsey A, Swan EK, Wilsey M. Herpes esophagitis with concomitant eosinophilic esophagitis in a child: a case report. Clin Pediatr (Phila) 2018;57:618-620.

17. Machicado JD, Younes M, Wolf DS. An unusual cause of odynophagia in a patient with eosinophilic esophagitis. Gastroenterology 2014;147:3738.

18. Lindberg GM, Van Eldik R, Saboorian MH. A case of herpes esophagitis after fluticasone propionate for eosinophilic esophagitis. Nat Clin Pract Gastroenterol Hepatol 2008;5:527-530. 